\title{
GROUPS WITH COMPLETE LATTICE OF NEARLY NORMAL SUBGROUPS
}

\author{
Maria DE FALCO and Carmela MUSELLA
}

\begin{abstract}
A subgroup $H$ of a group $G$ is said to be nearly normal in $G$ if it has finite index in its normal closure in $G$. A well-known theorem of B.H. Neumann states that every subgroup of a group $G$ is nearly normal if and only if the commutator subgroup $G^{\prime}$ is finite. In this article, groups in which the intersection and the join of each system of nearly normal subgroups are likewise nearly normal are considered, and some sufficient conditions for such groups to be finite-by-abelian are given.
\end{abstract}

\section{Introduction}

A subgroup $H$ of a group $G$ is said to be nearly normal in $G$ if it has finite index in its normal closure $H^{G}$. A famous theorem of B.H. Neumann [4] states that every subgroup of a group $G$ is nearly normal if and only if the commutator subgroup $G^{\prime}$ of $G$ is finite. The set $n n(G)$ of all nearly normal subgroups of a group $G$ is a sublattice of the subgroup lattice $\mathfrak{L}(G)$ of $G$; some properties of the lattice $n n(G)$ have recently been studied (see [1]). On the other hand it is easy to show that, for an arbitrary group $G, n n(G)$ need not be a complete sublattice of $\mathfrak{L}(G)$.

The aim of this article is to prove that if $G$ is a group such that $n n(G)$ is a complete sublattice of $\mathfrak{L}(G)$, then all subgroups of $G$ are nearly normal, provided that $G$ is rich in some sense of nearly normal subgroups. This latter assumption cannot be avoided since in any direct product of infinite simple groups every nearly normal subgroup is normal.

The corresponding problem for the lattice $a n(G)$ of all almost normal subgroups (i.e. subgroups with only finitely many conjugates) of a group $G$ was considered by L.A. Kurdachenko and S. Rinauro [3].

2000 Mathematics Subject Classification: 20E15, 20F24.

Servicio de Publicaciones. Universidad Complutense. Madrid, 2002 
Most of our notation is standard and can be found in [5].

\section{Statements and Proofs}

Let $\mathfrak{L}$ be a lattice. A sublattice $\mathcal{E}$ of $\mathfrak{L}$ is said to be $\wedge$-complete (respectively $\vee$-complete) if for every collection $\left(x_{i}\right)_{i \in I}$ of elements of $\mathcal{E}, \bigwedge_{i \in I} x_{i}$ (respectively $\bigvee_{i \in I} x_{i}$ ) belongs to $\mathcal{E}$. The sublattice $\mathcal{E}$ is called complete if it is both $\wedge$-complete and $\vee$-complete.

If $G$ is a group, a normal subgroup $N$ of $G$ is said to be $G$-hypofinite if it has a descending series with finite factors consisting of normal subgroups of $G$. Clearly every normal subgroup of a hypofinite group $G$ is $G$-hypofinite; in particular, this holds when $G$ is a residually finite group.

Lemma 1. Let $G$ be a group such that $n n(G)$ is a $\wedge$-complete sublattice of $\mathfrak{L}(G)$, and let $N$ be a $G$-hypofinite normal subgroup of $G$. If $x$ is an element of $G$ such that $\langle x, N\rangle$ is nearly normal and either $x$ has finite order or the coset $x N$ has infinite order, then $\langle x\rangle$ is a nearly normal subgroup of $G$.

Proof. Let

$$
N=N_{0}>N_{1}>\cdots>N_{\tau}=\{1\}
$$

be a descending series of normal subgroups of $G$ with finite factors. Assume by contradiction that the subgroup $\langle x\rangle$ is not nearly normal in $G$, and let $\alpha \leq \tau$ be the least ordinal such that $\langle x\rangle N_{\alpha}$ is not nearly normal. Clearly $\alpha>0$. Suppose first that $\alpha$ is not a limit ordinal, so that $\langle x\rangle N_{\alpha-1}$ is a nearly normal subgroup of $G$, and hence the index $\left|\langle x\rangle^{G} N_{\alpha-1}:\langle x\rangle N_{\alpha-1}\right|$ is finite. As the group $N_{\alpha-1} / N_{\alpha}$ is finite, it follows that also $\langle x\rangle N_{\alpha}$ has finite index in its normal closure. This contradiction shows that $\alpha$ is a limit ordinal. If the element $x$ has finite order, there exists an ordinal $\delta<\alpha$ such that $\langle x\rangle \cap N_{\alpha}=\langle x\rangle \cap N_{\delta}$, and so

$$
\langle x\rangle N_{\alpha}=\bigcap_{\delta \leq \beta<\alpha}\langle x\rangle N_{\beta}
$$


Since $n n(G)$ is a $\wedge$-complete sublattice of $\mathfrak{L}(G)$, it follows that also $\langle x\rangle N_{\alpha}$ is nearly normal in $G$, a contradiction. Suppose finally that the coset $x N$ has infinite order, so that $\langle x\rangle \cap N=\{1\}$ and also in this case

$$
\langle x\rangle N_{\alpha}=\bigcap_{\beta<\alpha}\langle x\rangle N_{\beta}
$$

is a nearly normal subgroup of $G$. This last contradiction completes the proof of the lemma.

Corollary 2. Let $G$ be a periodic hypofinite group such that $n n(G)$ is a complete sublattice of $\mathfrak{L}(G)$. Then the commutator subgroup $G^{\prime}$ of $G$ is finite.

Proof. Since $G$ is hypofinite, it follows from Lemma 1 that every cyclic subgroup of $G$ is nearly normal. Then all subgroups of $G$ are nearly normal, and so $G^{\prime}$ is finite by Neumann's theorem.

Recall that the $F C$-centre of a group $G$ is the subgroup consisting of all elements of $G$ with finitely many conjugates, and that a group $G$ is said to be an $F C$-group if it coincides with its $F C$-centre. It is well known that the commutator subgroup of any $F C$-group is locally finite. Moreover if $x$ is an element of an arbitrary group $G$ such that the cyclic subgroup $\langle x\rangle$ is nearly normal, then $\langle x\rangle^{G} /\langle x\rangle_{G}$ is finite (see [2], Lemma 2.1 ), and, in particular, $x$ belongs to the $F C$-centre of $G$.

Corollary 3. Let $G$ be a non-periodic group such that $n n(G)$ is a complete sublattice of $\mathfrak{L}(G)$. If the commutator subgroup $G^{\prime}$ of $G$ is periodic and $G$-hypofinite, then $G^{\prime}$ is finite.

Proof. It follows from Lemma 1 that every infinite cyclic subgroup of $G$ is nearly normal. On the other hand, $G$ can be obviously generated by its elements of infinite order, so that $G$ is an $F C$-group and hence also its finite cyclic subgroups are nearly normal. Therefore every subgroup of $G$ is nearly normal, and $G^{\prime}$ is finite by Neumann's theorem.

Another easy consequence of Lemma 1 is that if $G$ is a periodic nilpotent group such that $n n(G)$ is a complete sublattice of $\mathfrak{L}(G)$, then $G$ has finite commutator subgroup. This cannot be extended to the case of periodic hypercentral groups, as the consideration of locally dihedral 2 -group shows. 
Theorem 4. Let $G$ be a finitely generated hyper-(abelian or finite) group such that $n n(G)$ is a $\wedge$-complete sublattice of $\mathfrak{L}(G)$. Then the factor group $G / Z(G)$ is finite.

Proof. Assume by contradiction that $G$ is not central-by-finite. Then $G$ has an homomorphic image $\bar{G}$ such that $\bar{G} / Z(\bar{G})$ is infinite but all proper homomorphic images of $\bar{G}$ are central-by-finite (see [5], Lemma 6.17). Replacing $G$ by $\bar{G}$ it can be assumed without loss of generality that every proper homomorphic image of $G$ is central-by-finite. If $G$ is polycyclicby-finite, it is well known that each subgroup of $G$ is intersection of subgroups of finite index (see for instance [8], p.18), so that $\mathfrak{L}(G)=$ $n n(G)$ and $G / Z(G)$ is finite. This contradiction shows that $G$ is not polycyclic-by-finite, and in particular $G$ has no finite non-trivial normal subgroups. Then $G$ contains an abelian non-trivial normal subgroup $A$; as $G / A$ is central-by-finite, the group $G$ is metabelian-by-finite, and so also residually finite (see [5] Part 2, Theorem 9.51). Moreover, the elements of finite order of $G / A$ form a finite subgroup $T / A$. Since $G$ is not polycyclic-by-finite, the factor group $G / A$ is infinite and so $T$ is properly contained in $G$. Let $x$ be any element of the set $G \backslash T$. Then the coset $x A$ has infinite order, and hence $\langle x\rangle$ is a nearly normal subgroup of $G$ by Lemma 1, so that $x$ belongs to the $F C$-centre of $G$. Therefore $G=\langle G \backslash T\rangle$ is a finitely generated $F C$-group, and $G / Z(G)$ is finite. This contradiction completes the proof.

In the statement of Theorem 4 the hypothesis that the sublattice $n n(G)$ of $\mathfrak{L}(G)$ is $\wedge$-complete cannot be replaced by the assumption that $n n(G)$ is a $\vee$-complete sublattice of $\mathfrak{L}(G)$; to see this, it is enough to consider any polycyclic-by-finite group with infinite commutator subgroup. It is more interesting to note that there exists a periodic reduced metabelian group $G$ such that the sublattice $n n(G)$ of $\mathfrak{L}(G)$ is $\vee$-complete but not $\wedge$-complete. In fact let $p$ be any prime number, and consider the groups

$$
A=D r_{n \in N} A_{n} \quad \text { and } \quad X=D r_{q \neq p} X_{q},
$$

where each $A_{n}$ has order $p$ and $\left|X_{q}\right|=q$ for every prime number $q \neq p$. There exists a split extension $G$ of $A$ by $X$ such that $A$ is the unique minimal normal subgroup of $G$, and in particular $C_{G}(A)=A$ (see [5] Part 1, p.152). If $H$ is any nearly normal subgroup of $G$, then $A$ is 
contained in $H^{G}$, and hence the index $|A: A \cap H|$ is finite; it follows that, if the subgroup $J$ is the join of a collection of nearly normal subgroups of $G$, then $|A J: J|$ is finite and so $J$ is nearly normal in $G$. Therefore $n n(G)$ is a $\vee$-complete sublattice of $\mathfrak{L}(G)$. On the other hand, each $A_{n}$ is not nearly normal in $G$, but it can be realized as intersection of subgroups of finite index of $A$, so that the sublattice $n n(G)$ of $\mathfrak{L}(G)$ is not $\wedge$-complete.

We shall say that a group $G$ is a $Z A F$-group if it has an ascending normal series whose factors are finite or central in $G$. Such groups are clearly rich of nearly normal subgroups. Note also that the class of $Z A F$-groups contains all hyperfinite groups and all hypercentral groups. Our main result deals with $Z A F$-groups for which the lattice of nearly normal subgroups is complete.

Lemma 5. Let $G$ be a hypofinite $Z A F$-group such that $n n(G)$ is a $\wedge$ complete sublattice of $\mathfrak{L}(G)$. If $x$ is an element of $G$ having finitely many conjugates, then the subgroup $\langle x\rangle$ is nearly normal in $G$.

Proof. Assume by contradiction that the lemma is false, and let

$$
\{1\}=G_{0}<G_{1}<\ldots<G_{\tau}=G
$$

be an ascending normal series whose infinite factors are central. Consider the least ordinal $\alpha \leq \tau$ for which $G_{\alpha}$ contains an element $y$ with finitely many conjugates in $G$ such that the index $\left|\langle y\rangle^{G}:\langle y\rangle\right|$ is infinite. Clearly $\alpha$ is not a limit ordinal and $y$ has infinite order. If also the coset $y G_{\alpha-1}$ has infinite order, by hypothesis $G_{\alpha} / G_{\alpha-1}$ is contained in $Z\left(G / G_{\alpha-1}\right)$, so that $\left\langle y, G_{\alpha-1}\right\rangle$ is a normal subgroup of $G$, and it follows from Lemma 1 that $\langle y\rangle$ is nearly normal in $G$. This contradiction shows that $y G_{\alpha-1}$ must have finite order, so that $y^{m} \in G_{\alpha-1}$ for some positive integer $m$. Thus the cyclic subgroup $\left\langle y^{m}\right\rangle$ is nearly normal in $G$, and hence $\left\langle y^{m}\right\rangle^{G} /\left\langle y^{m}\right\rangle_{G}$ is finite, so that $\left\langle y^{k}\right\rangle$ is normal in $G$ for some $k>0$. As $y$ has only finitely many conjugates in $G$, it follows that $\langle y\rangle$ is a nearly normal subgroup of $G$, a contradiction. The lemma is proved.

Recall that a subgroup $H$ of a group $G$ is almost normal if it has finitely many conjugates in $G$, or equivalently if the normalizer $N_{G}(H)$ has finite index in $G$. 
Theorem 6. Let $G$ be a hypofinite $Z A F$-group such that $n n(G)$ is a complete sublattice of $\mathfrak{L}(G)$. Then the commutator subgroup $G^{\prime}$ of $G$ is finite.

Proof. Assume by contradiction that $G^{\prime}$ is infinite, and let

$$
\{1\}=G_{0}<G_{1}<\cdots<G_{\tau}=G
$$

be an ascending normal series whose factors are either finite or central. Since $n n(G)$ is a complete sublattice of $\mathfrak{L}(G)$, it follows from Neumann's theorem that $G$ contains an element $g$ such that the subgroup $\langle g\rangle$ is not nearly normal; thus the conjugacy class of $g$ is infinite by Lemma 5 . Let $\alpha \leq \tau$ be the least ordinal such that $G_{\alpha}$ contains an element $x$ having infinitely many conjugates in $G$. Clearly $\alpha$ is not a limit ordinal, and all subgroups of $G_{\alpha-1}$ are nearly normal in $G$. Put $X=\left\langle x, G_{\alpha-1}\right\rangle$. As $G_{\alpha} / G_{\alpha-1}$ is either finite or central in $G$, the group $X^{G} / X_{G}$ is finite, and in particular $X$ is almost normal and nearly normal in $G$. Application of Lemma 1 yields that $x$ has infinite order and $x^{m} \in G_{\alpha-1}$ for some positive integer $m$. In particular, $\left\langle x^{m}\right\rangle$ is nearly normal in $G$, and so $\langle x\rangle /\langle x\rangle_{G}$ is finite. The group $G_{\alpha-1}$ is finite-by-abelian by Neumann's theorem, so that $\langle x\rangle \cap G_{\alpha-1}^{\prime}=\{1\}$. Let $M$ be a subgroup of $G_{\alpha-1}$ containing $G_{\alpha-1}^{\prime}$ which is maximal with respect to the condition $\langle x\rangle \cap M=\{1\}$. Since $M$ is nearly normal in $G$, the index $\left|M^{G}: M\right|$ is finite, so that $\langle x\rangle \cap M^{G}=\{1\}$ and $M=M^{G}$ is a normal subgroup of $G$. Let $T / M$ be the subgroup consisting of all elements of finite order of the abelian group $G_{\alpha-1} / M$. The coset $x M$ has infinite order, so that $\langle x\rangle \cap T=\{1\}$ and $T=M$; thus $G_{\alpha-1} / M$ is a torsion-free abelian group. If $H_{1} / M$ and $H_{2} / M$ are subgroups of $G_{\alpha-1} / M$ such that $H_{1} \cap H_{2}=M$, from the equality $\langle x\rangle \cap M=\{1\}$ it follows that either $\langle x\rangle \cap H_{1}=\{1\}$ or $\langle x\rangle \cap H_{2}=\{1\}$, so that either $H_{1}=M$ or $H_{2}=M$. Therefore $G_{\alpha-1} / M$ has rank 1 . As $x^{m}$ belongs to $G_{\alpha-1}$, the coset $x^{m} M$ lies in $C / M=C_{G_{\alpha-1} / M}(x M)$ and so $G_{\alpha-1} / C$ is periodic. Let $y$ be any element of $G_{\alpha-1}$; then $y^{k} \in C$ for some positive integer $k$, and hence

$$
M=\left[y^{k} M, x M\right]=[y M, x M]^{k},
$$

so that $[y M, x M]=M$. It follows that $C=G_{\alpha-1}$, and so the group $X / M$ is abelian and $\langle x\rangle \cap X^{\prime}=\{1\}$. If $K / X^{\prime}$ is the subgroup consisting of all elements of finite order of the abelian group $X / X^{\prime}$, it follows that 
$\langle x\rangle \cap K=\{1\}$. As $K$ is characteristic in $X$, it is normal in $N_{G}(X)$, and hence $K$ is almost normal in $G$. Moreover, $K \cap G_{\alpha-1}$ is a nearly normal subgroup of $G$, so that $\left(K \cap G_{\alpha-1}\right)^{G} / X^{\prime}$ is a periodic subgroup of $G_{\alpha-1} / X^{\prime}$ and hence $K \cap G_{\alpha-1}=\left(K \cap G_{\alpha-1}\right)^{G}$ is normal in $G$. Clearly $G_{\alpha-1}$ has finite index in $X$, so that $K \cap G_{\alpha-1}$ has finite index in $K$, and so also the index $\left|K^{G}: K\right|$ is finite. Thus $\langle x\rangle \cap K^{G}=\{1\}$. Since $X / K$ is a torsion-free abelian group, if $u$ is any element of $N_{G}(X)$ such that $\left[x^{m} K, u K\right]=1$, we have also that $[x K, u K]=1$. On the other hand, $x^{m}$ has finitely many conjugates in $G$, so that the conjugacy class of $x K$ in $N_{G}(X) / K$ is also finite, and hence $\langle x, K\rangle$ is an almost normal subgroup of $G$, and so the index $\left|\langle x\rangle^{G} K^{G}:\langle x\rangle K^{G}\right|$ is finite as $\langle x\rangle /\langle x\rangle_{G}$ is finite. Application of Lemma 1 yields now that the subgroup $\langle x\rangle$ is nearly normal in $G$, and so the conjugacy class of $x$ is finite. This contradiction completes the proof of the theorem.

We observe finally that the lattice of nearly normal subgroups of the wreath product $G=\mathbb{Z}\left(p^{\infty}\right) \curlywedge \mathbb{Z}_{n}$ (where $p$ is any prime number and $n \geq 2)$ is not a $\vee$-complete sublattice of $\mathfrak{L}(G)$; on the other hand, $n n(G)$ is obviously $\wedge$-complete, since $G$ satisfies the minimal condition on subgroups.

\section{References}

[1] M. De Falco, C. Musella, "Some lattice properties of nearly normal subgroups", Quaderni di Matematica, 8 (2001), 87-100.

[2] S. Franciosi, F. de Giovanni, "Groups satisfying the minimal condition on certain non-normal subgroups", Proceedings of Groups-Korea 1994", de Gruyter, Berlin, (1995), 107-118.

[3] L.A. Kurdachenko, S. Rinauro, "Intersection and join of almost normal subgroups", Comm. Algebra, 23 (1995), 1967-1974.

[4] B.H. Neumann, "Groups with finite classes of conjugate subgroups", Math. Z., 63 (1955), 76-96.

[5] D.J.S. Robinson, "Finiteness Conditions and Generalized Soluble Groups", Springer, Berlin (1972).

[6] D.J.S. Robinson, "A Course in the Theory of Groups", Springer-Verlag, New York (1982).

[7] R. Schmidt, "Subgroup Lattices of Groups", de Gruyter, Berlin (1994). 
[8] D. Segal, "Polycyclic Groups", Cambridge University Press, Cambridge, (1983)

Dipartimento di Matematica e Applicazioni Università degli studi di Napoli Federico II

Complesso Universitario di Monte S. Angelo

Via Cintia

I 80126, Napoli, (Italy)

E-mail: musella@matna2.dma.unina.it

E-mail: defalco@matna2.dma.unina.it

Recibido: 21 de Mayo de 2001

Revisado: 13 de Diciembre de 2001 